\title{
Primeiro registro de Lepidiella Enderlein (Diptera, Psychodidae, Psychodinae) no Brasil e descrição de uma espécie nova
}

\author{
Freddy Bravo \\ Departamento de Ciências Biológicas, Universidade Estadual de Feira de Santana. Avenida Universitária, 44031-460 Feira de \\ Santana, Bahia, Brasil. E-mail: fbravo@uefs.br
}

\begin{abstract}
First record of Lepidiella Enderlein (Diptera, Psychodidae, Psychodinae) from Brazil and description of a new species. Lepidiella spinosa sp. nov. from São Paulo state, southeastern Brazil, is described and illustrated. It is the first record of Lepidiella Enderlein, 1937 from Brazil.

KEY WORDS: Lepidiella spinosa, neotropics.
\end{abstract}

RESUMO. Neste trabalho descreve-se Lepidiella spinosa sp. nov., uma nova espécie de Psychodidae proveniente São Paulo, sudeste do Brasil é descrita e ilustrada. Este é o primeiro registro de Lepidiella Enderlein, 1937 no Brasil.

PALAVRAS CHAVE: Lepidiella ornata, região neotropical.

O gênero Lepidiella Enderlein, 1937 inclui as espécies que eram tratadas no gênero Syntomoza Enderlein, 1937, uma vez que o nome Syntomoza não é mais válido em Psychodidae (Collantes \& Hodkinson 2003). Lepidiella é um gênero neotropical, sendo conhecidas nove espécies (ENDERELein 1937, RapP 1945, SATChell 1955, Quate 1996, 1999, Collantes \& Martinez-Ortega 1997, 1998), nenhuma do Brasil: L. amaliae (Collantes \& MartinezOrtega, 1997), L. cervi (Satchell, 1955), L. lanuginosa Enderlein, 1937, L. matagalpensis (Collantes \& Martinez-Ortega, 1998), L. monteverdica (Quate, 1996), L. niveitarsis (Enderlein, 1937), L. pickeringi (Quate, 1999), L. albipeda (Rapp, 1945), L. zumbadoi (Quate, 1996). Para todas as espécies de Lepidiella têm sido descritos os machos, exceto para L. amaliae que é conhecida apenas por fêmeas.

Em recente visita ao Museu de Zoologia da Universidade de São Paulo (MZUSP), encontrou-se alguns espécimes de Lepidiella, coletados na Estação Biológica da Boracéia, São Paulo, Sudeste do Brasil. Neste trabalho, é descrita uma espécie nova de Lepidiella, sendo desta maneira, a primeira espécie desse gênero registrada no Brasil.

Todos os espécimes foram tratados com solução aquosa de hidróxido de potássio $(\mathrm{KOH})$ e montados em lâmina permanente sob bálsamo de canadá. Os exemplares estão depositados no Museu de Zoologia da Universidade de São Paulo, São Paulo, Brasil. Segue-se o sistema para as veias alares proposto por Colless \& McAlpine (1991) e as demais terminologias seguem McAlpine (1981).

Chave para machos do gênero Lepidiella Enderlein 1. Escapo com lobo apical na margem interna ...................... 2 Escapo com margem interna sem lobo apical
2. Largura do apódema edeagal igual à largura do gonocoxito

Apódema edeagal mais estreito que a largura do gonocoxito 4

3. Gonóstilo com o mesmo comprimento do gonocoxito; parâmeros paralelos L. pickeringi

Gonóstilos com o dobro do comprimento do gonocoxito; parâmeros convergentes L. albipeda

4. Comprimento do escapo igual a 2 vezes o comprimento do pedicelo L. cervi

Comprimento do escapo igual a 3 vezes o comprimento do pedicelo L. monteverdica

5. Largura do apódema edeagal igual à largura do gonocoxito

Largura do apódema menor que a largura do gonocoxito .... 8

6. Primeiro flagelômero fusiforme, $1 \mathrm{vez}$ o comprimento do pedicelo

Primeiro flagelômero cilíndrico, 1,5 vezes o comprimento do pedicelo L. niveitarsis

7. Margem anterior da asa convexa; gonocoxito $1 / 3$ do comprimento do gonóstilo L. hansoni

Margem anterior da asa reta; gonocoxito $1 / 2$ o comprimento do gonóstilo L. matagalpemsis

8. Escapo com comprimento igual ao comprimento do pedicelo; forquilha $R_{2}-R_{3}$ anterior ao ápice da veia $C u A$ L. lanuginosa

Escapo 2,5 vezes o comprimento do pedicelo; forquilha $\mathrm{R}_{2}-\mathrm{R}_{3}$ posterior ao ápice da veia $\mathrm{CuA}$. L. spinosa sp. nov. 


\section{Lepidiella spinosa sp. nov.}

\section{Figs $1-7$}

Material-tipo. Brasil, São Paulo: Estação Biológica da Boracéia, holótipo macho, 31.VIII.1973, F. Val leg. (MZUSP). Alótipo fêmea, mesma localidade, data de coleta e coletor do holótipo (MZUSP). Parátipos, 4 machos, mesma localidade, data de coleta e coletor do holótipo (MZUSP).

Etimologia. Do latim spinosa, espinho, refere-se ao aspecto posterior do edeago, semelhante a um conjunto de espinhos.

Holótipo macho. Comprimento do corpo, desde o início do tórax até o final do abdome 2,1 mm. Cabeça subcircular, sutura interocular com formato de $\mathrm{V}$ invertido; cornícula ausente; ponte ocular de quatro facetas de largura, separadas entre si por espaço igual a dois diâmetros de faceta. Antena incompleta no exemplar estudado; escapo subcilíndrico; pedicelo menor subesférico (Fig. 2); flagelômeros subcilíndricos (Fig. 2); ascóides digitiformes (Fig. 2). Palpo maxilar com quatro segmentos; comprimento relativo dos segmentos do palpo: 1,0:1,7:2,0:2,2 (Fig. 3). Comprimento da asa, $2,0 \mathrm{~mm}$; largura máxima, $0,7 \mathrm{~mm}$; Rs não pectinada; $\mathrm{R}_{2+3}$ não unida a Rs; $\mathrm{R}_{4}$ terminando no ápice; $\mathrm{R}_{5}$ não unida a $\mathrm{R}_{4}$; $\mathrm{M} 3$ não unida a $\mathrm{M}_{1+2}$ (Fig. 1). Cercos, gonocoxitos e gonóstilos com pilosidade (Figs 4-6). Ápice do esternito $10 \mathrm{com}$ micropilosidade (Fig. 6). Tergito 9 subquadrado (Fig. 6). Tergito 10 fundido ao esternito 10, com micropilosidade apical (Fig. 6). Cercos longos, digitiformes (Figs 4, 6), com grupo de aproximadamente nove tenáculas (Fig. 4). Placa pós-hipandrial retangular (Fig. 5). Gonocoxitos subcilíndricos; gonóstilos sub-triangular, com a base mais larga e ápice estreito (Fig. 5). Edeago simétrico com formato de $\mathrm{V}$, ápice terminando em ponta; dois pares de parâmeros, o externo maior que o interno (Fig. 5). Apódema ejaculador longo e estreito (Figs 4, 5). Apódema gonocoxal curto.

Fêmea. Semelhante ao macho exceto pelas características descritas a seguir. Comprimento do corpo, desde o início do tórax até o final do abdome 2,2 $\mathrm{mm}$. Comprimento relativo dos segmentos do palpo: 1,0:1,4:1,2:1,9. Comprimento da asa, 2,4 mm; largura máxima, 0,8 $\mathrm{mm}$. Placa subgenital sub-triangular com apófise bilobada (Fig. 7). Cercos compridos, duas vezes o comprimento da placa sub-genital.

Comentários. Os espécimes de Lepidiella podem ser facilmente reconhecidos porque a veia longitudinal $R_{4}$ termina no ápice da asa. Nenhum outro gênero neotropical de Psychodinae apresenta essa característica. Outro caráter que tem sido mencionado como importante para a definição de Lepidiella, é a presença de uma estrutura multilobada na parte posterior da cabeça dos machos, estrutura que é denominada por alguns autores de língua inglesa como "allurement organ" (QuATE 1996, 1999), "sensory organs" (Quate 1963) ou "cornicula" (SATchell 1955, Duckhouse 1974, Collantes \& Martinez-Ortega 1998). Neste trabalho foi adotado o nome de cornícula para essa estrutura cefálica, por ser um termo descritivo.

A presença de cornículas multilobadas têm sido observada apenas nos machos de Lepidiella entre os gêneros neotropicais.
L. amaliae é conhecida apenas por fêmeas, sendo, desta maneira, desconhecida a presença de cornícula nesta espécie.

Dois outros caracteres, não exclusivos do gênero Lepidiella, podem ser usados na identificação desse gênero: Rs não pectinada e presença de várias tenáculas no ápice do cerco do macho.

Dos quatro caracteres acima citados para a identificação de Lepidiella, somente três foram observados na espécie nova L. spinosa: $\mathrm{R}_{4}$ terminando no ápice da asa, Rs não pectinada $\mathrm{e}$ presença de várias tenáculas no cerco do macho. Não foi observada a cornícula multilobada nos exemplares macho da espécie nova. Assume-se que a ausência da cornícula em L. spinosa seja uma condição derivada nessa espécie.

Lepidiella spinosa sp. nov. e L. amaliae diferenciam-se, principalmente, pelo formato da placa subgenital da fêmea. Em vista ventral, o comprimento da placa subgenital de $L$. spinosa sp. nov. é quase da largura do esternito 8, enquanto que em L. amaliae a placa subgenital é menor que a largura do esternito 8. O formato do lobo da placa subgenital também é diferente nas duas espécies, sendo subretangular em L. amaliae e mais abaulado, semelhante a um feijão, em L. spinosa sp. nov.

\section{AGRADECIMENTOS}

Ao CNPq (470754/2003-6) e FAPESB (PPP) pelo apóio financeiro. $\mathrm{O}$ autor recebe uma bolsa de produtividade do $\mathrm{CNPq}$ (307357/2003-1).

\section{REFERÊNCIAS BIBLIOGRÁFICAS}

Collantes, F. \& E. Martinez-Ortega. 1997. Syntomoza amaliae, a new apecies of moth- fly (Diptera, Psychodidae) from Nicaragua. Studies on Neotropical Fauna and Environment, Lisse, 32: 239-243.

Collantes, F. \& E. Martinez-Ortega. 1998. Syntomoza matagalpensis, a new moth-fly from Nicaragua (Diptera, Psychodidae). Aquatic Insects, Lisse, 20: 209-213.

Collantes, F. \& I.D. Hodkinson. 2003. The genus Syntomoza, a homonymy in Hemiptera and Diptera. A proposal of a new taxonomic status and check-list of related bibliography. Boletín de la Asociación Española de Entomologia, Madrid, 27: 231-232.

Colles, D.H. \& D.K. McAlpine. 1991. Diptera, p. 717-786. In: CSIRO. The insects of Australia. Victoria, Melbourne University Press, 1137p.

Duckhouse, D.A. 1974. Redescriptiuon of the Neotropical Psychodidae (Diptera, Nematocera) described by Rapp and Curran. Journal of Entomology (B), London, 43: 55-62.

Enderlein, G. 1937. Klassifikation der Psychodiden (Dipt.). Deutsche entomologische Zeitschrift, Berlin, 1936: 81-112. McAlpine, J.F. 1981. Morphology and terminology: adults, p. 963. In: J.F. McAlpine; B.V. Peterson; G.E. Shewell.; H.J.Teskey.; J.R. Vocкеroth,. \& D.M. Wood (Eds). Manual of Neartic Diptera. Ottawa, Research Branch, Agriculture Canada, Monograph 27, vol. 1, 674p. 




Figuras 1-7. Lepidiella spinosa sp. nov. (1-6) Macho, holótipo: (1) asa; (2) base da antena, pedicelo, escapo e três primeiros flagelômeros; (3) palpo maxilar; (4-7) terminália masculina: (4) lateral; (6) dorsal; (7) ventral; (7) terminália feminina, ventral da fêmea, alótipo.

Quate, L.W. 1963. Review of G. Enderlein's non holartic genera of Psychodidae and description of a new species (Diptera). Transactions of the Royal Entomological Society of London, London, 115: 181-196.

Quate, L.W. 1996. Preliminary taxonomy of Costa Rican Psycho- didae (Diptera) exclusive of Phlebotominae. Revista de Biología Tropical, San José, 44 (Suppl. 1): 1-81.

Quate, L.W. 1999. Taxonomy of neotropical Psychodidae (Dipter) 3. Psychodines of Barro Colorado Island and San Blas, Panama. Memoirs Entomology International, Gain- 
sesville, 14: 409-441.

Rapp. W.F. 1945. New Psychodidae from Barro Colorado Island. Journal of the New York Entomological Society, New York, 53: 309-311; Plate 3.
SATChelL, G.H. 1955. XI. Two new subgenera of Psychodidae (Díptera) from Jamaica, with descriptions of five new species. Annals and Magazine of Natural History. London, 12: 85-93.

Recebido em 13.IX.2004; aceito em 09.VI.2005. 